\title{
Clinical evaluation of a novel fractional radiofrequency based device for hair growth stimulation
}

\author{
Ines Verner* \\ The Clinic of Dermatology and Aesthetics, Tel Aviv, Israel
}

\begin{abstract}
Background and objective: Androgenetic alopecia is a common disorder affecting both males and females. Treatments range from topical or oral medication and energy based devices to hair transplantation. The purpose of this study was to evaluate the efficacy and safety of a novel fractional radiofrequency (RF) device (HairLux, Innogen Technologies Ltd., Yokneam, Israel), to prevent hair loss and induce hair growth.

Materials and methods: 25 patients received ten fractional RF treatments every two weeks, and were followed up 2 months after the last treatment. All patients were evaluated by global photography. In 10 patients blinded manual hair counts were performed. Results: Patients demonstrated less hair shedding, fuller hair, and faster hair growth. There was an average increase of $31.6 \%$ in hair density (based on hair counts) and 18\% increase in hair shaft thickness. All subjects tolerated the treatments well.
\end{abstract}

Conclusion: The HairLux device is effective and safe for hair growth stimulation in AGA. Ten treatment sessions are recommended to maximize results.

\section{Introduction}

Typically presenting with a progressive thinning, miniaturization, and loss of hair on the scalp, patterned hair loss or androgenetic alopecia is the most common cause of hair loss in both the men and women, affecting approximately $85 \%$ of males and $50 \%$ of females over 50 years of age. The hallmark of androgenetic alopecia is a miniaturization of terminal pigmented anagen hairs, culminating in the expression of fine hypopigmented vellus hairs [1,2]. In essence, hair loss occurs when there is a change in the anagen/telogen ratio. The shortening of anagen and lengthening of the telogen phase leads to a reduction in hair density, visually appreciable as thinning and increasingly sparse hair on the scalp.

The mechanism of female pattern hair loss remains unclear and here, the effect of DHT (dihydrotestosterone) appears to be less important and is thought to play less of a role in the development of this disorder in females. In contrast however, male pattern hair loss is clearly mediated by DHT, produced from testosterone by $5 a$-reductase. Both male and female hair loss types result in vellus hair transformation, hair follicle miniaturization, diminution of shaft diameter, a shortening of anagen growth cycles and lengthening of telogen resting phase, as well as hair follicle apoptosis; these changes are thought to be related to the specific action of androgens [2,3]. Other mechanisms that help maintain anagen and hair shaft differentiation during hair follicle development include growth factors such as IGF-1 which prolongs anagen and induces hair shaft differentiation. While downregulation of IGF-1 in the dermal papillae induces entry from anagen (growth) into the catagen (involution) phase. Other growth factors which are produced following RF and which play a role in the prolongation of anagen are VEGF (Vascular Endothelial Growth Factor), FGF 5 (Fibroblast Growth factor) and FGF 7 [4].

Many different treatment modalities and techniques including both surgical and non-surgical approaches have been employed to help sustain the hair and/or help achieve hair growth in hair loss patients, albeit with varying degrees of therapeutic success. Pharmaceutical agents such as minoxidil, finasteride (competitive inhibitors of type II $5 \alpha$-reductase), and dutasteride (competitive inhibitor of type I and II $5 \alpha$-reductase) are being readily used in androgenetic alopecia, and are first-line treatments that have shown to be somewhat effective in stemming hair loss and maintaining still viable hair [5,1]. Energybased systems including laser and light sources are enjoying an uptick in interest in androgenetic alopecia such as low-level laser therapy or LLLT (red or near infrared light) [1,3,6-15], most peculiarly due to the well-documented and yet still unclear hair growth effect they seem to have, known as paradoxical hypertrichosis [16-21]. LLLT appears to modulate cellular function including levels of prostaglandins, cytokines, and tumor necrosis factor, activate dormant hair follicles and helps to increase blood flow to the hair follicles [7]. Similarly, RF based devices have also been shown to be successful in promoting hair growth, thought to occur through the induction of growth factors in the papillary dermis [4].

One commonality among professional energy-based devices, is that after creating micro wounds in the skin, they induce a wound healing mechanism, one that leads to positive responses in local tissues such as hair growth. Previous studies have shown that wound healing is a key factor in stimulating hair follicles and hair growth [22-

Correspondence to: Ines Verner, Verner Clinic Tel Aviv, 39 Jerusalem St., pob 39, Kiriat Ono 55423, Israel, Tel: 972-3-6353183; Fax: 972-3-5341381; ines.verner@ gmail.com

Key words: hair, alopecia, androgenetic alopecia, radiofrequency, topical therpy, hair growth stimulation

Received: August 20, 2017; Accepted: September 21, 2017; Published: September 25, 2017 
24]. Although the mechanism of this hair growth remains partially unknown, hair growth has been seen to occur following the induction of wound healing mechanisms. The aim of this study was to investigate the efficacy and safety of an RF device to achieve hair growth.

\section{Materials and methods}

\section{Study design}

This was a prospective, open label, single center, clinical study.

Twenty-five healthy patients aged 19 to 73 (18 females, 7 males) with variable grades of AGA according to the Hamilton Norwood, and Ludwig pattern hair loss scales were included and scheduled to receive ten treatments at two-week intervals. The study was approved by an Institutional Review Board and all study participants provided written informed consent prior to the initiation of the trial. Global photography was performed in all patients at baseline, at every second treatment and 2 months after the last treatment. Comparisons were made at the end of the study. In 10 patients (aged 38 to 57) dermatoscopic images were taken and sent to an independent laboratory (TrichoLAB, Warsaw, Poland) for measurement and blinded evaluation of manual hair counts, hair density (the average number of hair in $\mathrm{cm}^{2}$ ) and hair follicle thickness (the average hair shaft thickness in $\mathrm{cm}^{2}$ ). Treatment safety was evaluated by the frequency and severity of adverse events reported throughout the clinical trial. Patients also completed a self-assessment questionnaire to help further evaluate improvements achieved as well as overall patient satisfaction and comfort from treatment.

Treatment safety was evaluated by the frequency and severity of adverse events reported throughout the clinical trial.

\section{Device description}

The HairLux device (Innogen Technologies Ltd., Yokneam, Israel) is a professional hair growth stimulation fractional RF technology. The tabletop device is equipped with a fractional RF applicator (handpiece). The system employs an innovative RF distribution mechanism, based on a combination of monopolar and bipolar RF technologies, with the therapeutic advantages of both treatment modalities. The comb-like RF applicator tip consists of an array of $6 \times 6$ pins, totaling 36 pins. Each pin operates as an independent electrode, and has an independent electrical switcher, enabling each electrode to change polarity (+/-). During treatment, each of the electrodes, or pins, transmits the electrical RF current in a uniform pattern to the target area on the scalp, creating localized stimulation which promotes hair growth.

The key advantage of this innovative technology is the high control over the RF current pathway which leads to fast treatments with high efficacy, high safety profile and no pain during the treatment., The pins function similarly to comb teeth and part the patient's hair, ensuring that the RF current is homogenously distributed and delivered to the scalp. The HairLux system produces RF energy in $460 \mathrm{KHz}$ with equivalent energy of up to $20 \mathrm{~mJ}$ per pin for 36 electrodes. Treatments are characteristically painless, ensuring a pleasant treatment experience for the patient.

\section{Results}

Twenty five healthy study subjects having the typical clinical picture of androgenetic alopecia respective to their gender were included in this experimental clinical study.

Of the 25 study participants who were enrolled, baseline data and study evaluation measurements were made and collected for ten patients (female: $\mathrm{n}=8$; male: $\mathrm{n}=2$ ). Two patients dropped out as they could not commit to the study protocol.

Twenty-three patients who completed the study protocol showed overall less hair shedding, faster hair growth, fuller hair and more hair volume after 10 treatments and at 2 months follow up.

Trichoscopy manual hair count results of all ten patients demonstrated a mean $31.6 \%$ improvement in hair counts, with an average hair count in the frontal area increasing from 136 hairs $/ \mathrm{cm}^{2}$ to 179 hairs $/ \mathrm{cm}^{2}$. Hair thickness measurements demonstrated an $18 \%$ increase in hair shaft thickness. The mean hair thickness in the frontal area increased from $48.4 \mu \mathrm{m}$ to $57.1 \mu \mathrm{m}$ (vellus hair: $<30 \mu \mathrm{m}$; intermediate hair: 30-50 $\mu \mathrm{m}$; terminal hair: $>50 \mu \mathrm{m})$.

An increased density and thickness of the hair, verified with hair count data, led to a significantly positive cosmetic outcome in all study participants. The procedure was also found to be safe, with no serious or lasting adverse events. While some of the patients reported only a mild tingling during treatment and mild scalp sensitivity lasting from a few hours up to 24 hours post treatment, none of the patients reported of any pain or discomfort, and all tolerated the treatment sessions well. No serious adverse events were observed.

In the self-assessed questionnaires, 13 and 10 subjects reported to be either very satisfied or satisfied, respectively. All 23 subjects would recommend the procedure.

Figures 1-3 illustrate the positive cosmetic impact seen from baseline after multiple treatments with the HairLux device.

\section{Discussion}

Androgenetic alopecia (pattern hair loss) is very challenging to treat and although many different treatment modalities are available (topical minoxidil, oral finasteride, hair transplantation and low laser light therapy) results are variable and mostly unsatisfactory.
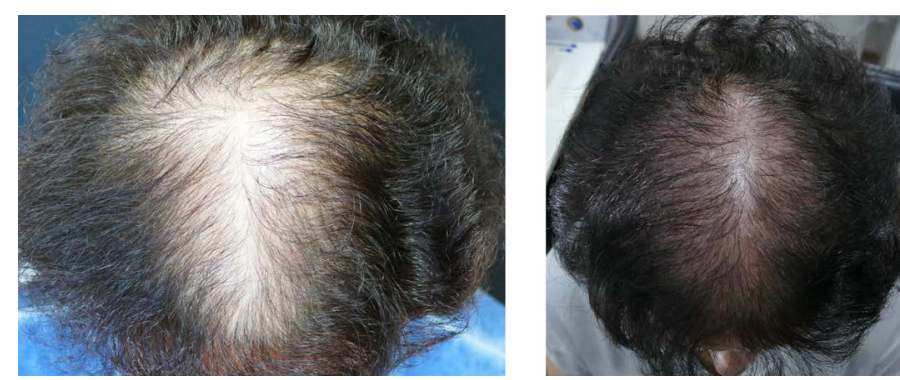

Figure 1. A 54 year old patient with Female Pattern Hair Loss before and after 10 HairLux treatments
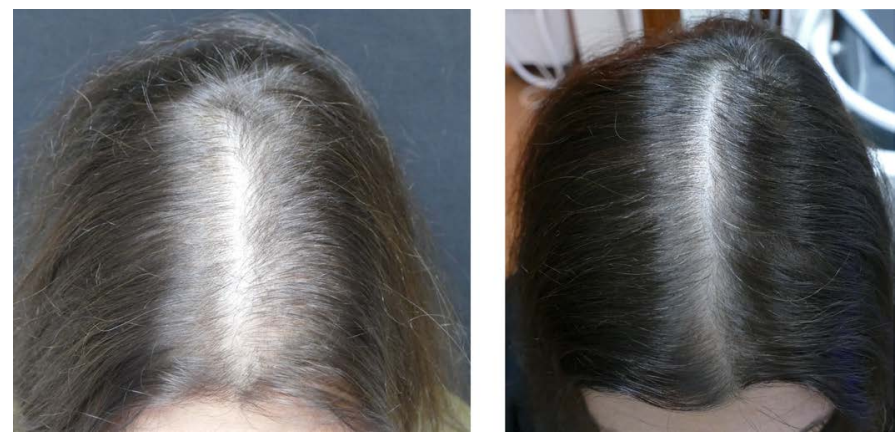

Figure 2. A 46 year old patient with Female Pattern Hair Loss before and after 10 HairLux treatments 
In pattern hair loss, hair follicle structures are preserved while disruption of hair growth (anagen) and/or prolongation of the resting phase (telogen) due to inadequate hair follicle stem cell (HFSC) stimulation, lead to hair loss. Therefore treatments that prolong or induce the anagen phase may be helpful.

New hair growth requires entry of hair follicles from the telogen (resting) into the anagen (growth) phase. This involves a two-step activation process: First the secondary hair germ precursors (present at the base of the follicle) proliferate and only second and after this the hair follicle bulge stem cells proliferate. This two-step activation happens during late telogen to early anagen.

One of the earliest molecular pathways for the initiation of this two-step activation and later hair shaft formation is the $\mathrm{Wnt} / \mathrm{b}$-catenin pathway.

B-catenin is a downstream mediator of Wnt signaling and is present in the dermal papilla during anagen whereas the absence of b-catenin leads to premature catagen induction.

Interestingly the Wnt/b-catenin pathway is also one of the initial molecular responses to injury and is activated by wounding. Dermal papillae cells react to wounding by the production of mediators (proteins) that pass signals by the Wnt pathway. In this pathway, there is a (paracrine) cross talk between the dermal papillae cells (secondary hair germ) and bulge cells.

After skin wounding, Wnt signal activates stem cells to repair tissue and enhance formation of new epithelia including hair. Hence, Wnt signaling not only leads to complete tissue regeneration but also to new hair follicle formation.

Radiofrequency (RF) is a high frequency alternating electric current with a frequency between $3 \mathrm{kHz}-300 \mathrm{GHz}$. It's first use in medicine was in 1928 by Dr. Cushing and Dr. Bovie.They used pulsed RF energy and were able to remove tumors while minimizing hemorrhagic complications. The first tissue tighteningand rejuvenation device that used RF energy was a monopolar RF device introduced in 2003 and the first bipolar fractional RF device for skin resurfacing and rejuvenation was introduced in 2009.

Since then many other RF devices have been introduced into the market with nowadays a plethora of devices for tissue tightening and rejuvenation.

The reason RF technology has become one of the most popular technologies for skin rejuvenation is its high safety profile and efficacy. Moreover RF has no preference for melanin or other "colored" molecules or structures making it "color blind". Thus it can be safely used also in the darker skin types [22-24].

Suggested mechanisms for the induction of hair growth by radiofrequency include the induction of cytokines and growth factors that are associated with hair growth such as keratinocyte growth factor, IGF-1 (Insulin like growth gactor-1). Yoon in 2011 [4] showed upregulation of IGF-1, a potent stimulator of hair follicle growth and VEGF (vascular endothelial growth factor) in human dermal papillae cells after RF exposure together with hair shaft elongation [4].

Fractional RF devices which are used for skin resurfacing and collagen remodeling induce new collagen formation in the dermis. This entirely new extracellular matrix in the dermal papillae with robust neocollagenesis, increase in hyaluronic acid formation as well as elastogenesis results in a healthier scalp and improved anchorage of the hair in the follicles [25]. Leading to longer hair growth and less hair loss.
The HairLux RF device effectively stimulates the scalp and induces hair growth by triggering growth factor production, induction of a very mild wound healing response and the induction of neocollagenesis. RF also increases blood circulation via VEGF resulting in higher and more effective tissue perfusion.

In this study, we used the HairLux device to induce significant hair growth on the scalp. Patients demonstrated a faster hair growth, fuller hair, and more hair volume after 8 to 10 treatments, with $31.6 \%$ and $18 \%$ showing improvements in hair counts and hair thickness, respectively. None of the patients reported any discomfort from treatment and patients were very pleased with the cosmetic results. The HairLux device offers a safe and effective treatment alternative for patients suffering from androgenetic alopecia.

\section{Compliance with ethical standards}

Funding: Innogen Technologies supplied the device for the study

Informed consent: All patients signed a written informed consent form prior to study initiation according to ethical regulations.

\section{References}

1. Kim WS, Lee HI, Lee JW, Lim YY, Lee SJ, et al. (2011). Fractional photothermolysis laser treatment of male pattern hair loss. Dermatol Surg 37: 41-51. [Crossref]

2. Paus R, Olsen EA, Messenger AG (2008) Hair growth disorder. In: Fitzpatrick's dermatology in general medicine. (7thedtn). McGraw-Hill, New York. p. 753-77.

3. Avci P, Gupta GK, Clark J, Wikonkal N, Hamblin MR (2014) Low- Level Laser (Light) Therapy (LLLT) for treatment of hair loss. Lasers Surg Med 46:144-151. [Crossref]

4. Yoon SY, Kim KT, Jo SJ, Cho AR, Jeon SI, et al. (2011) Induction of hair growth by insulin-like growth factor-1 in $1,763 \mathrm{MHz}$ radiofrequency-irradiated hair follicle cells PloS one 6: e28474.[Crossref]

5. Khandpur S, Suman M, Reddy BS (2002) Comparative efficacy of various treatmen regimens for androgenetic alopecia in men. J Dermatol 29: 489-98. [Crossref]

6. Avram MR, Leonard RT Jr, Epstein ES, Williams JL, Bauman AJ (2007) The current role of laser/light sources in the treatment of male and female pattern hair loss. $J$ Cosmet Laser Ther 9: 27-28. [Crossref]

7. Avram MR, Rogers NE (2009) The use of low-level light for hair growth: Part I. $J$ Cosmet Laser Ther 11: 110-117. [Crossref]

8. Hamblin MR (2006) Mechanisms of low level light therapy. Proc SPIE 6140: 1-12.

9. Jimenez JJ, Wikramanayake TC, Bergfeld W, Hordinsky M, Hickman JG, et al. (2014) Efficacy and safety of a low-level laser device in the treatment of male and female pattern hair loss: a multicenter, randomized, sham device-controlled, double-blind study. Am J Clin Dermatol 15: 115-127. [Crossref]

10. Lanzafame RJ, Blanche RR, Chiacchierini RP, Kazmirek ER, Sklar JA (2014) The growth of human scalp hair in females using visible red light laser and LED sources. Lasers Surg Med 46: 601-607. [Crossref]

11. Leavitt M, Charles G, Heyman E, Michaels D (2009) HairMax Laser Comb laser phototherapy device in the treatment of male androgenetic alopecia: A randomized, double-blind, sham device-controlled, multicentre trial. Clin Drug Invest 29: 283-292. [Crossref]

12. Lee GY, Lee SJ, Kim WS (2011) The effect of a $1550 \mathrm{~nm}$ fractional erbium-glass laser in female pattern hair loss. J Eur Acad Dermatol Venereol 25: 1450-1454. [Crossref]

13. Satino JL, Markou M (2003) Hair regrowth and increased hair tensile strength using the HairMax LaserComb for low-level laser therapy. Int J Cosmet Surg Aesth Dermatol 5: 113-117.

14. Stillman L (2010) Reply to: The use of low-level light for hair growth: Part I. J Cosmet Laser Ther 12: 116. [Crossref]

15. Yamazaki M, Miura Y, Tsuboi R, Ogawa H (2003) Linear polarized infrared irradiation using Super Lizer is an effective treatment for multiple-type alopecia areata. Intl $J$ Dermatol 42: 738-740. [Crossref]

16. Alajlan A, Shapiro J, Rivers JK, MacDonald N, Wiggin J, et al. (2005). Paradoxical hypertrichosis after laser epilation. J Am Acad Dermatol 53: 85-88. [Crossref] 
17. Bernstein EF (2005) Hair growth induced by diode laser treatment. Dermatol Surg 31: 584-586. [Crossref]

18. Bouzari N, Firooz AR (2006) Lasers may induce terminal hair growth. Dermatol Surg 32: 460. [Crossref]

19. Kontoes P1, Vlachos S, Konstantinos M, Anastasia L, Myrto S (2006) Hair induction after laser-assisted hair removal and its treatment. J Am Acad Dermatol 54: 64-67. [Crossref]

20. Lolis MS, Marmur ES (2006) Paradoxical effects of hair removal systems: A review. $J$ Cosmet Dermatol 5: 274-276. [Crossref]

21. Willey A, Torrontegui J, Azpiazu J, Landa N (2007) Hair stimulation following laser and intense pulsed light photo- epilation: Review of 543 cases and ways to manage it. Lasers Surg Med 39: 297-301. [Crossref]
22. Chuong CM (2007) Regenerative biology: new hair from healing wounds. Nature 17: 265-266. [Crossref]

23. Jiang S, Zhao L, Teklemariam T, Hantash BM (2010) Small cutaneous wounds induce telogen to anagen transition of murine hair follicle stem cells. J Dermatol Sci 60: 143150. [Crossref]

24. Levy V, Lindon C, Zheng Y, Harfe BD, Morgan BA (2007) Epidermal stem cells arise from the hair follicle after wounding. FASEB J 21: 1358-1366. [Crossref]

25. Zelickson BD, Kist D, Bernstein E, Brown DB, Ksenzenko S, et al. (2004). Histological and ultrastructural evaluation of the effects of a radiofrequency-based nonablative dermal remodeling device: a pilot study. Arch Dermatol 140: 204-209.[Crossref]

Copyright: (C2017 Verner I. This is an open-access article distributed under the terms of the Creative Commons Attribution License, which permits unrestricted use, distribution, and reproduction in any medium, provided the original author and source are credited. 